NBER WORKING PAPER SERIES

\title{
MODELING BOND YIELDS IN FINANCE AND MACROECONOMICS
}

\author{
Francis X. Diebold \\ Monika Piazzesi \\ Glenn D. Rudebusch \\ Working Paper 11089 \\ http://www.nber.org/papers/w11089
NATIONAL BUREAU OF ECONOMIC RESEARCH
1050 Massachusetts Avenue
Cambridge, MA 02138
January 2005

The views expressed in this paper do not necessarily reflect those of the Federal Reserve Bank of San Francisco. We thank our colleagues and, in particular, our many co-authors. The views expressed herein are those of the author(s) and do not necessarily reflect the views of the National Bureau of Economic Research.

(C) 2005 by Francis X. Diebold, Monika Piazzesi, and Glenn D. Rudebusch. All rights reserved. Short sections of text, not to exceed two paragraphs, may be quoted without explicit permission provided that full credit, including $(\odot)$ notice, is given to the source. 
Modeling Bond Yields in Finance and Macroeconomics

Francis X. Diebold, Monika Piazzesi, and Glenn D. Rudebusch

NBER Working Paper No. 11089

January 2005

JEL No. G1, E4, E5

\begin{abstract}
From a macroeconomic perspective, the short-term interest rate is a policy instrument under the direct control of the central bank. From a finance perspective, long rates are risk-adjusted averages of expected future short rates. Thus, as illustrated by much recent research, a joint macro-finance modeling strategy will provide the most comprehensive understanding of the term structure of interest rates. We discuss various questions that arise in this research, and we also present a new examination of the relationship between two prominent dynamic, latent factor models in this literature: the Nelson-Siegel and affne no-arbitrage term structure models.
\end{abstract}

\author{
Francis X. Diebold \\ Department of Economics \\ University of Pennsylvania \\ Philadelphia, PA 19104 \\ and NBER \\ fdiebold@ sas.upenn.edu \\ Monika Piazzesi \\ Graduate School of Business \\ University of Chicago \\ Chicago, IL 60637 \\ and NBER \\ monika.piazzesi@gsb.uchicago.edu \\ Glenn D. Rudebusch \\ Economic Research \\ Federal Reserve Bank of San Francisco \\ 101 Market Street \\ San Francisco, CA 94105 \\ glenn.rudebusch@sf.frb.org
}


From a macroeconomic perspective, the short-term interest rate is a policy instrument under the direct control of the central bank, which adjusts the rate to achieve its economic stabilization goals. From a finance perspective, the short rate is a fundamental building block for yields of other maturities, which are just risk-adjusted averages of expected future short rates. Thus, as illustrated by much recent research, a joint macro-finance modeling strategy will provide the most comprehensive understanding of the term structure of interest rates. In this paper, we discuss some salient questions that arise in this research, and we also present a new examination of the relationship between two prominent dynamic, latent factor models in this literature: the Nelson-Siegel and affine no-arbitrage term structure models.

\section{Questions about Modeling Yields}

(1) Why use factor models for bond yields? The first problem faced in term structure modeling is how to summarize the price information at any point in time for the large number of nominal bonds that are traded. In fact, since only a small number of sources of systematic risk appear to underlie the pricing of the myriad of tradable financial assets, nearly all bond price information can be summarized with just a few constructed variables or factors. Therefore, yield curve models almost invariably employ a structure that consists of a small set of factors and the associated factor loadings that relate yields of different maturities to those factors. Besides providing a useful compression of information, a factor structure is also consistent with the celebrated "parsimony principle," the broad insight that imposing restrictions - even those that are false and may degrade in-sample fit - often helps both to avoid data mining and to produce good forecasting models. For example, an unrestricted 
Vector Autoregression (VAR) provides a very general linear model of yields, but the large number of estimated coefficients renders it of dubious value for prediction (Diebold and Calin Li, 2005). Parsimony is also a consideration for determining the number of factors needed, along with the demands of the precise application. For example, to capture the time series variation in yields, one or two factors may suffice since the first two principal components account for almost all (99\%) of the variation in yields. Also, for forecasting yields, using just a few factors may often provide the greatest accuracy. However, more than two factors will invariably be needed in order to obtain a close fit to the entire yield curve at any point in time, say, for pricing derivatives.

(2) How should bond yield factors and factor loadings be constructed? There are a variety of methods employed in the literature. One general approach places structure only on the estimated factors. For example, the factors could be the first few principal components, which are restricted to be mutually orthogonal, while the loadings are relatively unrestricted. Indeed, the first three principal components typically closely match simple empirical proxies for level (e.g., the long rate), slope (e.g., a long minus short rate), and curvature (e.g., a midmaturity rate minus a short and long rate average). A second approach, which is popular among market and central bank practitioners, is a fitted Nelson-Siegel curve (introduced in Charles Nelson and Andrew Siegel, 1987). As described by Diebold and Li (2005), this representation is effectively a dynamic three-factor model of level, slope, and curvature. However, the Nelson-Siegel factors are unobserved, or latent, which allows for measurement error, and the associated loadings have plausible economic restrictions (forward rates are always positive, and the discount factor approaches zero as maturity increases). A third approach is the no-arbitrage dynamic latent factor model, which is the model of choice 
in finance. The most common subclass of these models postulates flexible linear or affine forms for the latent factors and their loadings along with restrictions that rule out arbitrage strategies involving various bonds.

(3) How should macroeconomic variables be combined with yield factors? Both the Nelson-Siegel and affine no-arbitrage dynamic latent factor models provide useful statistical descriptions of the yield curve, but they offer little insight into the nature of the underlying economic forces that drive its movements. To shed some light on the fundamental determinants of interest rates, researchers have begun to incorporate macroeconomic variables into these yield curve models.

For example, Diebold, Rudebusch, and S. Boragan Aruoba (2005) provide a macroeconomic interpretation of the Nelson-Siegel representation by combining it with VAR dynamics for the macroeconomy. Their maximum likelihood estimation approach extracts three latent factors (essentially level, slope, and curvature) from a set of 17 yields on U.S. Treasury securities and simultaneously relates these factors to three observable macroeconomic variables (specifically, real activity, inflation, and a monetary policy instrument).

The role of macroeconomic variables in a no-arbitrage affine model is explored by several papers. In Piazzesi (2005), the key observable factor is the Federal Reserve's interest rate target. The target follows a step function or pure jump process, with jump probabilities that depend on the schedule of policy meetings and three latent factors, which also affect risk premiums. The short rate is modeled as the sum of the target and short-lived deviations from target. The model is estimated with high-frequency data and provides a new identification scheme for monetary policy. The empirical results show that relative to standard latent factor models using macroeconomic information can substantially lower pricing errors. In 
particular, including the Fed's target as one of four factors allows the model to match both the short and the long end of the yield curve.

In Andrew Ang and Piazzesi (2003) and Ang, Sen Dong, and Piazzesi (2004), the macroeconomic factors are measures of inflation and real activity. The joint dynamics of these macro factors and additional latent factors are captured by VARs. In Ang and Piazzesi (2003), the measures of real activity and inflation are each constructed as the first principal component of a large set of candidate macroeconomic series, to avoid relying on specific macro series. Both papers explore various methods to identify structural shocks. They differ in the dynamic linkages between macro factors and yields, discussed further below.

Finally, Rudebusch and Tao Wu (2004a) provide an example of a macro-finance specification that employs more macroeconomic structure and includes both rational expectations and inertial elements. They obtain a good fit to the data with a model that combines an affine no-arbitrage dynamic specification for yields and a small fairly standard macro model, which consists of a monetary policy reaction function, an output Euler equation, and an inflation equation.

(4) What are the links between macro variables and yield curve factors? Diebold, Rudebusch, and Aruoba (2005) examine the correlations between Nelson-Siegel yield factors and macroeconomic variables. They find that the level factor is highly correlated with inflation, and the slope factor is highly correlated with real activity. The curvature factor appears unrelated to any of the main macroeconomic variables. Similar results with a more structural interpretation are obtained in Rudebusch and Wu (2004a); in their model, the level factor reflects market participants' views about the underlying or medium-term inflation target of the central bank, and the slope factor captures the cyclical response of the central bank, 
which manipulates the short rate to fulfill its dual mandate to stabilize the real economy and keep inflation close to target. In addition, shocks to the level factor feed back to the real economy through an ex ante real interest rate.

Piazzesi (2005), Ang and Piazzesi (2003) and Ang, Dong, and Piazzesi (2004) examine the structural impulse responses of the macro and latent factors that jointly drive yields in their models. For example, Piazzesi (2005) documents that monetary policy shocks change the slope of the yield curve, because they affect short rates more than long ones. Ang and Piazzesi (2003) find that output shocks have a significant impact on intermediate yields and curvature, while inflation surprises have large effects on the level of the entire yield curve. They also find that better interest rate forecasts are obtained in an affine model in which macro factors are added to the usual latent factors.

For estimation tractability, Ang and Piazzesi (2003) only allow for unidirectional dynamics in their arbitrage-free model, specifically, macro variables help determine yields but not the reverse. Diebold, Rudebusch, and Aruoba (2005) consider a general bidirectional characterization of the dynamic interactions and find that the causality from the macroeconomy to yields is indeed significantly stronger than in the reverse direction but that interactions in both directions can be important. Ang, Dong, and Piazzesi (2004) also allow for bidirectional macro-finance links but impose the no-arbitrage restriction as well, which poses a severe estimation challenge that is solved via Markov Chain Monte Carlo methods. The authors find that the amount of yield variation that can be attributed to macro factors depends on whether or not the system allows for bidirectional linkages. When the interactions are constrained to be unidirectional (from macro to yield factors), macro factors can only explain a small portion of the variance of long yields. In contrast, the bidirectional system 
attributes over half of the variance of long yields to macro factors.

(5) How useful are no-arbitrage modeling restrictions? The assumption of no arbitrage ensures that, after accounting for risk, the dynamic evolution of yields over time is consistent with the cross-sectional shape of the yield curve at any point in time. This consistency condition is likely to hold, given the existence of deep and well-organized bond markets. However, if the underlying factor model is misspecified, such restrictions may actually degrade empirical performance. (Of course, the ultimate goal is a model that is both internally consistent and correctly specified.) Ang and Piazzesi (2003) present some empirical evidence favorable to imposing no-arbitrage restrictions because of improved forecasting performance. As discussed below, this issue is worthy of further investigation.

(6) What is the appropriate specification of term premiums? With risk-neutral investors, yields are equal to the average value of expected future short rates (up to Jensen's inequality terms), and there are no expected excess returns on bonds. In this setting, the expectations hypothesis, which is still a mainstay of much casual and formal macroeconomic analysis, is valid. However, it seems likely that bonds, which provide an uncertain return, are owned by the same investors who also demand a large equity premium as compensation for holding risky stocks. Furthermore, as suggested by many statistical tests in the literature, these risk premiums on nominal bonds appear to vary over time, contradicting the assumption of risk-neutrality. To model these premiums, Ang and Piazzesi (2003) and Rudebusch and Wu (2004a, b) specify time-varying "prices of risk," which translate a unit of factor volatility into a term premium. This time variation is modeled using business cycle indicators such as the slope of the yield curve or measures of real activity. However, Diebold, Rudebusch, and Aruoba (2005) suggest that the importance of the statistical deviations from the expectations 
hypothesis may depend on the application.

\section{Example: An Affine Interpretation of Nelson-Siegel}

In this section, we develop a new example to illustrate several of the above issues, particularly the construction of yield curve factors and the imposition of the no-arbitrage restrictions. By showing how to impose no-arbitrage restrictions in a Nelson-Siegel representation of the yield curve, we outline a methodology to judge these restrictions. The Nelson-Siegel model is a popular model that performs well in forecasting applications, so it would be interesting to compare its accuracy with and without these restrictions (a subject of our ongoing research).

The 2-factor Nelson-Siegel model specifies the yield on a $\tau$-period bond as

$$
y_{t}^{(\tau)}=a_{\tau}^{N S}+b_{\tau}^{N S} \cdot x_{t}
$$

where $x_{t}$ is a 2-dimensional vector of latent factors (or state variables) and the yield coefficients depend only on the time to maturity $\tau$ :

$$
\begin{aligned}
& a_{\tau}^{N S}=0 \\
& b_{\tau}^{N S}=\left[\begin{array}{ll}
1 & \frac{1-\exp (-k \tau)}{k \tau}
\end{array}\right]^{\top} .
\end{aligned}
$$

The two coefficients in $b_{\tau}^{N S}$ give the loadings of yields on the two factors. The first loading is unity, so the first factor operates as a level shifter and affects yields of all maturities one-forone. The second loading goes to one as $\tau \rightarrow 0$ and goes to zero as $\tau \rightarrow \infty$ (assuming $k>0$ ), so the second factor mainly affects short maturities and, hence, the slope. Furthermore, as maturity $\tau$ goes to zero, the yield in equation (1) approaches the instantaneous short rate of interest, denoted $r_{t}$, and, since the second component of $b_{\tau}^{N S}$ goes to 1 , the short rate is 
just the sum of the two factors,

$$
r_{t}=x_{t}^{1}+x_{t}^{2}
$$

and is latent as well. Finally, as in Diebold and Li (2005), we augment the cross-sectional equation (1) with factor dynamics; specifically, both components of $x_{t}$ are independent $\operatorname{AR}(1)$ 's:

$$
x_{t}^{i}=\mu_{i}+\rho_{i} x_{t-1}^{i}+v_{i} \varepsilon_{t}^{i}
$$

with Gaussian errors $\varepsilon_{t}^{i}, i=1,2$. Therefore, the complete Nelson-Siegel dynamic representation, (1), (2), (3), (5), has 7 free parameters: $k, \mu_{1}, \rho_{1}, v_{1}, \mu_{2}, \rho_{2}$, and $v_{2}$.

Consider now the 2-factor affine no-arbitrage term structure model. This model starts from the linear short rate equation (4); however, rather than postulating a particular functional form for the factor loadings as above, the loadings are derived from the short rate equation (4) and the factor dynamics (5) under the assumption of an absence of arbitrage opportunities. In particular, if there are risk-neutral investors, they are indifferent between buying a long bond that pays off $\$ 1$ after $\tau$ periods and an investment that rolls over cash at the short rate during those $\tau$ periods and has an expected payoff of $\$ 1$. Thus, risk-neutral investors would engage in arbitrage until the $\tau$-period bond price equals the expected rollover amount, so the yield on a $\tau$-period bond will equal the expected average future short rate over the $\tau$ periods (plus a Jensen's inequality term.) Risk-averse investors will need additional compensation for holding risky positions, but the same reasoning applies after correcting for risk premiums. Therefore, to make the Nelson-Siegel model internally consistent under the assumption of no-arbitrage, yields computed from expected average future short rates using (4) with the factor dynamics (5) must be consistent with the cross-sectional 
specification in equations (1) through (3).

To enforce this no-arbitrage internal consistency, we switch to continuous time and fix the sampling frequency so that the interval $[t-1, t]$ covers, say, one month. The continuous-time $\mathrm{AR}(1)$ process corresponding to (5) is

$$
d x_{t}^{i}=\kappa_{i}\left(\theta_{i}-x_{t}^{i}\right) d t+\sigma_{i} d B_{t}^{i}
$$

where $\kappa_{i}, \theta_{i}$ and $\sigma_{i}$ are constants and $B^{i}$ is a Brownian motion (which means that $d B^{i}$ is normally distributed with mean zero and variance $d t$ ). (In continuous time, the Nelson-Siegel has 7 parameters: $k, \kappa_{1}, \theta_{1}, \sigma_{1}, \kappa_{2}, \theta_{2}$, and $\left.\sigma_{2}.\right)$

We first consider the model with risk-neutral investors, which consists of the linear short rate equation (4) and the factor dynamics (6) and has 6 parameters: $\kappa_{1}, \theta_{1}, \sigma_{1}, \kappa_{2}, \theta_{2}$, and $\sigma_{2}$. Investors engage in arbitrage until the time- $t$ price $P_{t}^{(\tau)}$ of the $\tau$-bond is given by

$$
P_{t}^{(\tau)}=E_{t}\left(\exp \left(-\int_{t}^{t+\tau} r_{s} d s\right)\right) .
$$

This expectation can be computed by hand, since the short rate is the sum of two Gaussian $\mathrm{AR}(1)$ 's and is thus normally distributed. (The appendix details these calculations.) The resulting $\tau$-period yield is

$$
\begin{aligned}
y_{t}^{(\tau)} & =-\frac{\log P_{t}^{(\tau)}}{\tau} \\
& =a_{\tau}^{N A}+b_{\tau}^{N A} \cdot x_{t},
\end{aligned}
$$

with the no-arbitrage factor loadings given by

$$
b_{\tau}^{N A}=\left[\begin{array}{ll}
\frac{1-\exp \left(-\kappa_{1} \tau\right)}{\kappa_{1} \tau} & \frac{1-\exp \left(-\kappa_{2} \tau\right)}{\kappa_{2} \tau}
\end{array}\right]^{\top}
$$

The equations (4), (6), (8), and (9) constitute a canonical affine term-structure specification with two Gaussian factors. Intuitively, in the risk-neutral world, where yields are based 
only on expected future short rates, the cross-sectional factor-loading coefficients $b_{\tau}^{N A}$ are restricted to be functions of the time series parameters $\kappa_{1}$ and $\kappa_{2}$. The constant $a_{\tau}^{N A}$ absorbs any Jensen's inequality terms. In general, the Nelson-Siegel representation does not impose this dynamic consistency restriction because the loadings $b_{\tau}^{N S}$ are not related to the time series parameters from the $\mathrm{AR}(1)$. However, the no-arbitrage restriction can be applied to the Nelson-Siegel model under two conditions. First, let $\kappa_{1}$ go to zero and set $\kappa_{2}=k$, since for these parameter values, $b_{\tau}^{N A}=b_{\tau}^{N S}$. Second, it will have to be case that the constant $a_{\tau}^{N A}$, which embeds the Jensen's inequality terms, is close to zero for reasonable parameter values, i.e., $a_{\tau}^{N A} \approx a_{\tau}^{N S}=0$. (As a rule, macroeconomists often ignore Jensen's terms; however, with near-random walk components in the short rate process as $\kappa_{1}$ goes to zero, the Jensen's terms may be large, especially for long maturities $\tau$.)

Now consider the more general case of no-arbitrage with risk-averse investors. To accommodate departures from risk-neutrality, we parametrize the risk premiums used to adjust expectations. For example, suppose the pricing kernel solves

$$
\frac{d m_{t}}{m_{t}}=-r_{t} d t-\lambda_{t}^{1} d B_{t}^{1}-\lambda_{t}^{2} d B_{t}^{2}
$$

where

$$
\lambda_{t}^{i}=\lambda_{0}^{i}+\lambda_{1}^{i} x_{t}^{i}
$$

and $\lambda_{0}^{i}, \lambda_{1}^{i}$ are constants. The variables $\lambda_{t}^{i}$ are the prices of risk for each Brownian motion and are affine functions of the factors and so vary over time. The no-arbitrage factor loadings are given by

$$
b_{\tau}^{N A}=\left[\begin{array}{ll}
\frac{1-\exp \left(-\kappa_{1}^{*} \tau\right)}{\kappa_{1}^{*} \tau} & \frac{1-\exp \left(-\kappa_{2}^{*} \tau\right)}{\kappa_{2}^{*} \tau}
\end{array}\right]^{\top}
$$


where

$$
\kappa_{i}^{*}=\kappa_{i}+\sigma_{i} \lambda_{1}^{i}
$$

This 2-factor Gaussian model has 10 parameters $\lambda_{0}^{1}, \lambda_{1}^{1}, \lambda_{0}^{2}, \lambda_{1}^{2}, \kappa_{1}, \theta_{1}, \sigma_{1}, \kappa_{2}, \theta_{2}$, and $\sigma_{2}$. Now it is possible to pick the slope parameters, $\lambda_{1}^{i}$, so that the loadings, $b_{\tau}^{N A}$, equal the NelsonSiegel loadings, $b_{\tau}^{N S}$. The values for $\lambda_{1}^{i}$ that meet this condition are obtained by setting $\kappa_{1}^{*}=0$ and $\kappa_{2}^{*}=k$, and these imply that

$$
\lambda_{1}^{1}=-\frac{\kappa_{1}}{\sigma_{1}} \text { and } \lambda_{1}^{2}=\frac{k-\kappa_{2}}{\sigma_{2}}
$$

The constant terms in the market prices of risk are unrestricted, so we can set $\lambda_{0}^{1}=\lambda_{0}^{2}=0$. Again, it will have to be case that the Jensen's inequality terms should be close to zero, so $a_{\tau}^{N A} \approx a_{\tau}^{N S}=0$

\section{The Future}

The macro-finance term structure literature is in its infancy with many unresolved but promising issues to explore. For example, as suggested above, the appropriate specification for the time-series forecasting of bond yields is an exciting area for additional research, especially in a global context (Diebold, Li, and Vivian Yue 2005). In addition, the goal of an estimated no-arbitrage macro-finance model specified in terms of underlying preference and technology parameters (so the asset-pricing kernel is consistent with the macrodynamics) remains a major challenge. 


\section{REFERENCES}

Ang, Andrew and Piazzesi, Monika. "A No-Arbitrage Vector Autoregression of Term Structure Dynamics with Macroeconomic and Latent Variables." Journal of Monetary Economics, 2003, 50, pp. 745-787.

Ang, Andrew; Dong, Sen and Piazzesi, Monika. "No-Arbitrage Taylor Rules." Working paper, University of Chicago, 2004.

Diebold, Francis X. and Li, Calin. "Forecasting the Term Structure of Government Bond Yields." Forthcoming, Journal of Econometrics, 2005.

Diebold, Francis X.; Li, Calin and Yue, Vivian. "Modeling Term Structures of Global Bond Yields." Working paper, University of Pennsylvania, 2005.

Diebold, Francis X.; Rudebusch, Glenn D. and Aruoba, S. Boragan. "The Macroeconomy and the Yield Curve: A Dynamic Latent Factor Approach." Forthcoming, Journal of Econometrics, 2005.

Nelson, Charles R. and Siegel, Andrew. "Parsimonious Modeling of Yield Curves." Journal of Business, 1987, 60, pp. 473-489.

Piazzesi, Monika. "Bond Yields and the Federal Reserve." Forthcoming, Journal of Political Economy, 2005.

Rudebusch, Glenn D. and Wu, Tao. "A Macro-Finance Model of the Term Structure, Monetary Policy, and the Economy." Working paper, Federal Reserve Bank of San Francisco, 2004a. 
Rudebusch, Glenn D. and Wu, Tao. "The Recent Shift in Term Structure Behavior from a No-Arbitrage Macro-Finance Perspective." Working paper, Federal Reserve Bank of San Francisco, 2004b. 


\section{Appendix}

To derive the affine bond pricing formulas and yield curve equations, consider the case

with prices of risk $\lambda_{t}=\left[\begin{array}{ll}\lambda_{t}^{1} & \lambda_{t}^{2}\end{array}\right]^{\top}$. (Note that equation (9) can be obtained from (10) by setting the prices of risk to zero.) There are two ways to derive thes formulas. First, we can construct a risk-neutral probability measure under which the risk-neutral pricing formula (7) holds. Second, we can start from the Euler equation $E\left[d\left(m_{t} F_{t}\right)\right]=0$.

\section{Risk-neutral probability}

Under the risk-neutral probability measure, the process $B^{*}$ which solves $d B_{t}^{*}=d B_{t}+\lambda_{t} d t$ is a Brownian motion. By solving for $d B_{t}$ and inserting this expression into the $\operatorname{AR}(1)$ dynamics of the factors (6), we get

$$
\begin{aligned}
d x_{t}^{i} & =\kappa_{i}\left(\theta_{i}-x_{t}^{i}\right) d t+\sigma_{i}\left(d B_{t}^{* i}-\lambda_{t}^{i} d t\right) \\
& =\left(\kappa_{i} \theta_{i}-\kappa_{i} x_{t}^{i}-\sigma_{i} \lambda_{0}^{i}-\sigma_{i} \lambda_{1}^{i} x_{t}^{i}\right) d t+\sigma_{i} d B_{t}^{* i} \\
& =\left(\kappa_{i} \theta_{i}-\sigma_{i} \lambda_{0}^{i}-\left(\kappa_{i}+\sigma_{i} \lambda_{1}^{i}\right) x_{t}^{i}\right) d t+\sigma_{i} d B_{t}^{* i} \\
& =\left(\kappa_{i}+\sigma_{i} \lambda_{1}^{i}\right)\left(\frac{\kappa_{i} \theta_{i}-\sigma_{i} \lambda_{0}^{i}}{\left(\kappa_{i}+\sigma_{i} \lambda_{1}^{i}\right)}-x_{t}^{i}\right) d t+\sigma_{i} d B_{t}^{* i} \\
& =\kappa_{i}^{*}\left(\theta_{i}^{*}-x_{t}^{i}\right) d t+\sigma_{i} d B_{t}^{* i},
\end{aligned}
$$

where

$$
\begin{aligned}
\kappa_{i}^{*} & =\kappa_{i}+\sigma_{i} \lambda_{1}^{i} \\
\theta_{i}^{*} & =\frac{\kappa_{i} \theta_{i}-\sigma_{i} \lambda_{0}^{i}}{\kappa_{i}+\sigma_{i} \lambda_{1}^{i}}
\end{aligned}
$$

The price of the $\tau$-period bond is equal to

$$
P_{t}^{(\tau)}=E_{t}^{*}\left(\exp \left(-\int_{t}^{t+\tau} r_{s} d s\right)\right)
$$


where the expectation operator $E^{*}$ uses the risk-neutral probability measure. Since the vector $x=\left(x_{1}, x_{2}\right)^{\top}$ is Markov, this expectation is a function of the state today $x_{t}$. Thus, the bond price is a function

$$
P_{t}^{(\tau)}=F\left(x_{t}, \tau\right)
$$

of the state vector $x_{t}$ and time-to-maturity $\tau$. The Feynman-Kac formula says that $F$ solves the partial differential equation

$$
F_{t} r_{t}=-\frac{\partial F}{\partial \tau}+\sum_{i=1}^{2}\left[\frac{\partial F}{\partial x^{i}} \kappa_{i}^{*}\left(\theta_{i}^{*}-x_{t}^{i}\right)+\frac{1}{2} \frac{\partial^{2} F}{\partial x^{i 2}} \sigma_{i}^{2}\right]
$$

with terminal condition $F(x, 0)=1$.

We guess the solution

$$
F\left(x_{t}, \tau\right)=\exp \left(A(\tau)+B(\tau) \cdot x_{t}\right)
$$

which means that

$$
\begin{aligned}
\frac{\partial F}{\partial x^{i}} & =B_{i}(\tau) F \\
\frac{\partial^{2} F}{\partial x^{i 2}} & =B_{i}(\tau)^{2} F \\
\frac{\partial F}{\partial \tau} & =\left(A^{\prime}(\tau)+B^{\prime}(\tau) \cdot x_{t}\right) F .
\end{aligned}
$$

Insert these expressions into the partial differential equation and get

$$
\begin{aligned}
x_{t}^{1}+x_{t}^{2}= & -A^{\prime}(\tau)-B_{1}^{\prime}(\tau) x_{t}^{1}-B_{2}^{\prime}(\tau) x_{t}^{2} \\
& +\sum_{i=1}^{2}\left[B_{i}(\tau) \kappa_{i}^{*}\left(\theta_{i}^{*}-x_{t}^{i}\right)+\frac{1}{2} B_{i}(\tau)^{2} \sigma_{i}^{2}\right] .
\end{aligned}
$$


Matching coefficients results in

$$
\begin{aligned}
A^{\prime}(\tau) & =\sum_{i=1}^{2} B_{i}(\tau) \kappa_{i}^{*} \theta_{i}^{*}+\frac{1}{2} B_{i}(\tau)^{2} \sigma_{i}^{2} \\
1 & =-B_{1}^{\prime}(\tau)-B_{1}(\tau) \kappa_{1}^{*} \\
1 & =-B_{2}^{\prime}(\tau)-B_{2}(\tau) \kappa_{2}^{*} .
\end{aligned}
$$

The boundary conditions are

$$
\begin{aligned}
& A(0)=0 \\
& B(0)=0_{2 \times 1} .
\end{aligned}
$$

The solution to these ODE's are

$$
\begin{aligned}
& B_{1}(\tau)=\frac{\left(\exp \left(-\kappa_{1}^{*} \tau\right)-1\right)}{\kappa_{1}^{*}} \\
& B_{2}(\tau)=\frac{\left(\exp \left(-\kappa_{2}^{*} \tau\right)-1\right)}{\kappa_{2}^{*}} .
\end{aligned}
$$

We can plug these solutions into the yield equation

$$
\begin{aligned}
y_{t}^{(\tau)} & =-\frac{A(\tau)}{\tau}-\frac{B_{1}(\tau)}{\tau} x_{t}^{1}-\frac{B_{2}(\tau)}{\tau} x_{t}^{2} \\
& =a^{N A}(\tau)+b_{1}^{N A}(\tau) x_{t}^{1}+b_{2}^{N A}(\tau) x_{t}^{2}
\end{aligned}
$$

and get equations (9).

\section{Euler equation approach}

The Euler equation is

$$
P_{t}^{(\tau)}=E_{t}\left[\frac{m_{t+\tau}}{m_{t}}\right]
$$

and the instantaneous equation is

$$
E\left[d\left(m_{t} F_{t}\right)\right]=0
$$


The bond price is a function $F(x, \tau)$ and we can apply Ito's Lemma

$$
d F=\mu_{F} d t+\sigma_{F} d B_{t},
$$

where the drift and volatility of $F$ are given by

$$
\begin{aligned}
\mu_{F} & =-\frac{\partial F}{\partial \tau}+\sum_{i=1}^{2}\left[\frac{\partial F}{\partial x_{i}} \kappa_{i}\left(\theta_{i}-x^{i}\right)+\frac{1}{2} \frac{\partial^{2} F}{\partial x^{i 2}} \sigma_{i}^{2}\right] \\
\sigma_{F} & =\sum_{i=1}^{2} \frac{\partial F}{\partial x^{i}} \sigma_{i}
\end{aligned}
$$

Both $m_{t}$ and $F_{t}$ are Ito processes, so their product solves

$$
\begin{aligned}
d\left(m_{t} F_{t}\right)= & -r_{t} m_{t} F_{t} d t+m_{t} \mu_{t}^{F} d t-m_{t} \lambda_{t} \sigma_{t}^{F} d t \\
& -F_{t} m_{t} \lambda_{t} d B_{t}+m_{t} \sigma_{t}^{F} d B_{t}
\end{aligned}
$$

We use the Euler equation (19) and get

$$
\begin{aligned}
0 & =-r_{t} m_{t} F_{t}+m_{t} \mu_{t}^{F}-m_{t} \lambda_{t} \sigma_{t}^{F} \\
F_{t} r_{t} & =\left(-\frac{\partial F}{\partial \tau}+\sum_{i=1}^{2}\left[\frac{\partial F}{\partial x^{i}} \kappa_{i}\left(\theta_{i}-x_{t}^{i}\right)+\frac{1}{2} \frac{\partial^{2} F}{\partial x^{i 2}} \sigma_{i}^{2}\right]\right)-\sum_{i=1}^{2} \frac{\partial F}{\partial x^{i}} \sigma_{i} \lambda_{t}^{i}
\end{aligned}
$$

Again, guess the exponential-affine solution (16) and insert the expressions into (20), we get

$$
\begin{aligned}
x_{t}^{1}+x_{t}^{2}= & -A^{\prime}(\tau)-B_{1}^{\prime}(\tau) x_{t}^{1}-B_{2}^{\prime}(\tau) x_{t}^{2} \\
& +\sum_{i=1}^{2}\left[B_{i}(\tau) \kappa_{i}\left(\theta_{i}-x_{t}^{i}\right)+\frac{1}{2} B_{i}(\tau)^{2} \sigma_{i}^{2}\right] \\
& -\sum_{i=1}^{2} B_{i}(\tau) \sigma_{i}\left(\lambda_{0}^{i}+\lambda_{1}^{i} x_{t}^{i}\right) .
\end{aligned}
$$

Matching coefficients, we get the ordinary differential equations:

$$
\begin{aligned}
A^{\prime}(\tau) & =\sum_{i=1}^{2} B_{i}(\tau)\left(\kappa_{i} \theta_{i}-\sigma_{i} \lambda_{0}^{i}\right)+\frac{1}{2} B_{i}(\tau)^{2} \sigma_{i}^{2} \\
1 & =-B_{1}^{\prime}(\tau)-B_{1}(\tau)\left(\kappa_{1}+\sigma_{1} \lambda_{1}^{1}\right) \\
1 & =-B_{2}^{\prime}(\tau)-B_{2}(\tau)\left(\kappa_{2}+\sigma_{2} \lambda_{1}^{2}\right) .
\end{aligned}
$$


From this expression, we can see that we get the coefficients (17a) with risk neutral parameters

$$
\begin{aligned}
\kappa_{i}^{*} & =\kappa_{i}+\sigma_{i} \lambda_{1}^{i} \\
\kappa_{i}^{*} \theta_{i}^{*} & =\kappa_{i} \theta_{i}-\sigma_{i} \lambda_{0}^{i} \Longrightarrow \theta_{i}^{*}=\frac{\kappa_{i} \theta_{i}-\sigma_{i} \lambda_{0}^{i}}{\kappa_{i}+\sigma_{i} \lambda_{1}^{i}} .
\end{aligned}
$$

